\title{
Combining knowledge to generate novelty: A study of disclosed ideas for life science inventions
}

Taran Thune \& Magnus Gulbrandsen

TIK Center for Technology, Innovation and Culture

University of Oslo

taranmt@tik.uio.no

Final version published as:

Thune, Taran Mari \& Gulbrandsen, Magnus (2017). Combining knowledge to generate novelty: a study of disclosed ideas for life science inventions. European Journal of Innovation Management. ISSN 1460-1060. 20(3), s 446- 462. Doi: 10.1108/EJIM-11-2016-0114 


\begin{abstract}
Purpose: This paper investigates how a combination of diverse sources of knowledge is important for the generation of new ideas, and it addresses how institutional infrastructures and practices support integration of knowledge across organizations in medicine and life sciences.
\end{abstract}

Design: The paper investigates new product ideas that emerge from hospital and university employees and looks at the extent of interaction between clinical and scientific environments in the idea generation process. The paper utilizes a database of all new product ideas within life science that were filed in South-Eastern Norway in 2009 to 2011, including information about the individuals and teams that had been involved in disclosing these ideas. Interviews with inventors have also been carried out.

Findings: Interaction and integration across scientific and clinical domains are common and important for generating new product ideas. More than half of the disclosed life science ideas in the database come from groups representing multiple institutions with both scientific and clinical units or from individuals with multiple institutional affiliations. The interviews indicate that the infrastructure for cross-domain interaction is well-developed, particularly for research activities, which has a positive effect on invention.

Originality: The paper uses an original dataset of invention disclosures and investigates the hospitalscience interface, which is a novel setting for studies of inventive activities.

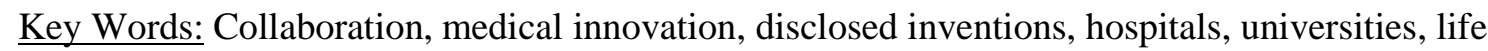
science commercialization 


\section{Introduction}

In medicine, which includes areas such as drugs, diagnostics and medical devices, inventions and innovations frequently originate in medical and scientific practice in public hospitals and universities, and often involve firms only in peripheral roles or later stages (Hopkins, 2006; Rosenberg et al., 2009; Morlacchi \& Nelson, 2011). However, traditional investigations of innovation typically look at how this takes place within firms and their greater networks, and there are comparatively few investigations of how innovative ideas related to what is often called "life sciences" may stem from other settings. In this paper, we ask how ideas with innovative potential emerge in the life sciences, which competencies these ideas are most likely to be based on, and how important the combination of different sources of knowledge is for generation and development of ideas. In addition, we analyze the practices that have been developed in a life science setting to facilitate integration across heterogeneous actors and knowledge bases in research and innovation.

Recent research on development of medical innovations has highlighted the importance of the interface between clinical and scientific knowledge for generation of novelty (Hopkins, 2006; Lander \& Atkinson-Grosjean, 2011; Lander, 2013; Ali \& Gittelman, 2016; Alkærsig \& Valentin, 2016). This interface is also important for dissemination and post-introductory improvements of new practices or products in medicine and healthcare (Consoli \& Mina, 2009; Nelson et al., 2011; Morlacchi \& Nelson, 2011). The combination of scientific and clinical competencies, which may be seen as two different "domains", seems to be related to quicker and more successful idea development and a higher rate of innovation.

There has consequently been a call for increased emphasis on so-called translational research aimed at combining "lab bench to bedside" perspectives within one project or organizational unit, or more generally research activities that aim to use basic biomedical discoveries for improving patient care. Increased interaction between biomedical research communities and clinical units has been seen as a central bridging instrument between different domains of medical knowledge (Lander \& AtkinsonGrosjean, 2011). Often this entails collaboration between hospitals and universities which is found to be common but also referred to as the "hidden research system" (Hicks \& Katz, 1996) as it is rarely investigated and because the outputs are seldom patents, spin-off companies or other easily measurable and visible phenomena. This paper contributes to the "hidden innovation" perspective by investigating whether collaboration between hospitals and universities also encompasses innovation activities and by investigating the relationship between integration of knowledge, research across domains and the generation of novelty. The processes and results of translational research are recent research topics, and questions have been raised about the effectiveness of the translational paradigm (Gulbrandsen et al., 2016). The paper aims to make a contribution here and to discuss insights from 
studies of translational research to the broader literature on the importance of knowledge integration for innovation.

Within innovation studies, several authors have made the argument that combination of diverse knowledge sources is a key prerequisite for innovation; since Schumpeter (1934) it is commonly argued that most innovations emerge out of novel combinations of established knowledge. Although integration of heterogeneous knowledge is important, it is also fundamentally problematic. The degree of novelty of knowledge involved and the complexity and radicalness of the innovation process are important features that determine knowledge integration processes and outcomes. These features also in turn influence the mechanisms that need to be in place to transfer and integrate knowledge across organizational boundaries (Hansen, 1999; Carlile, 2004).

The issue of knowledge integration across entities and its importance for innovation has been investigated at the meso (network) and micro levels. But there is less knowledge about the activities and practices of individuals in terms of how brokerage across domains occur and how knowledge that emerges at the interface is transformed and utilized in invention and innovation processes, or what Andersson \& Berggren (2011, p.91) refers to as "key practices of knowledge integration at a micro level". Such practices are expected to be highly context specific, and in this article the context is inventive activity in life science. This is a complex, multi-organizational setting encompassing vastly different activities. The study concerns new product ideas from hospital and university employees and analyzes interaction between clinical and scientific units in the idea development stage (inventive stage) of life science innovations disclosed by university and hospital staff. The analysis uses a similar perspective to Ali \& Gittelman (2016). Here it is assumed that cross-domain teams (consisting of staff employed in clinical and scientific units) and individuals that straddle domains represent key mechanisms for integration of scientific and clinical competencies, and that these individuals and teams are vital for generating innovations in medicine and health care.

But documentation of ideas and people and units that have generated ideas cannot tell us how integration occurs and why it is important for inventive work. To better understand this process, we have interviewed prolific life science inventors, i.e. individuals with multiple inventions. This approach is similar to Anderson \& Berggren (2011) who claimed that prolific inventors are of key importance for understanding knowledge generation and knowledge integration. In the inventor interviews we sought access to experiences about how knowledge from scientific and clinical environments is combined in different stages of the inventive process, illuminating knowledge integration practices in the particular context of specialist healthcare.

Before we make a more detailed description of the methods and data, we will make a brief review of literature of relevance for understanding how diverse knowledge is combined into innovative ideas in the healthcare setting. Based on the literature we formulate two propositions to guide the empirical 
analysis: that integration of knowledge from hospitals and academia is beneficial for invention, and that this integration needs to be institutionalized to support innovation in a wider sense. The results of the empirical study are then presented and discussed in light of existing literature, before conclusions and implications from the study are drawn.

\section{Combining knowledge for generating new ideas in life sciences}

Close linkages between science and the clinic have repeatedly been found to be important for health related research and innovation activities (Hicks and Katz, 1996; Lander and Atkinson-Grosjean, 2011; Hopkins, 2006). New knowledge and ideas for products and services emerge from a complex interplay between scientific units, clinical units and commercial organizations, and often also involve incremental technological and process improvements through learning in practice (Morlacchi \& Nelson, 2011). Several analysts emphasize that innovation processes in medicine and health care are "distributed", meaning that decentralized interaction across fields of science, organizations and sectors is a key feature of the problem-oriented learning processes that over time lead to development and use of new medical technologies, services or drugs (c.f. Blume, 1992; Rosenberg et al., 1995: Metcalfe et al., 2005; Ramlogan et al., 2007; Gittelman, 2016). In this distributed system, collaboration between research organizations and small high tech firms in the commercialization of knowledge is seen as particularly important for innovation, as impetus to medical innovations often comes from different part of the science system -not necessarily from life science. Several authors highlight how healthcare providers, clinical knowledge, and clinical practices are fundamental components of health innovation (Metcalfe et al., 2005; Morlacchi \&Nelson, 2011; Rosenberg, 2009; Nelson et al., 2011), but these actors' roles are not well understood.

Empirical research has shown that innovation within medicine is dependent upon knowledge generation occurring within the three separate domains - science, the clinic and technology - and the integration between them. Interaction between these domains occurs in different ways. Lander \& Atkinson-Grosjean (2011) discuss two common but very different "translations" between clinical, scientific and commercial organizational settings. They claim that that the translation of scientific knowledge to commercial use, the "commercialization pathway", has been the attention of most research on biomedical innovation as well as policy development. However, the science-clinical translation termed the "translational medicine pathway" is equally important and encompasses a conversion of scientific knowledge into clinical practice. This path is largely non-commercial and results in development of a large variety of new procedures, protocols, techniques, treatment options and more. The translational pathway does not only concern use of scientific knowledge in medical practice, but the process is recursive where problems, materials and insights from the clinic are brought to the scientific setting, and where shared resources across multiple sites are created. 
Resources such as knowledge, diagnostic tools, patient samples, research materials and analytical methods also move from the clinical domain to science.

According to Lander \& Atkinson-Grosjean (2011), translational science is performed in networks or communities where scientists and clinicians - as well as people with technological and administrative roles, patient advocates, and policy makers - "converge around a particular medical problem" which functions as a boundary object. Mina (2009) refers to these as micro-innovation systems, central for generation of novelty as well as dissemination and use of innovations. Several in-depth empirical studies of such micro-innovation systems can be found connected to particular medical problems or particular medical procedures or technologies (see Thune \& Mina, 2016 for a review of these studies). According to Nelson et al. (2011), progress in human medicine has been heavily dependent on development of new technological capabilities and scientific advances in fields outside of medicine. They argue that development of new diagnostic or therapeutic interventions is "inherently interdisciplinary" which requires integration of techno-scientific knowledge with knowledge of human medicine. Nelson et al. (2011) propose that the experimental learning that occurs within and connected to practice, in particular clinical practice, is an important mechanism for integration of diverse sets of knowledge, which over time leads to new inventions and innovations in human medicine.

A key finding in studies of medical technologies and disease areas is that boundaries between science, technology and medical practices are transient or porous (Lander \& Atkinson-Grosjean, 2011). Individuals and teams that act as boundary spanners are important for linking different domains and integrating knowledge across them, but the tools and technologies developed in these communities are themselves important bridging mechanisms. At the same time, the process of integrating scientific breakthroughs with medical practice to generate innovations is problematic and fraught with challenges, and most initiatives fail (at least in the short run) (Robertson, 2007; Hopkins et al., 2007). According to Robertson (2007) one important reason for the lack of impact (or lack of integration) of breakthrough scientific discoveries on medical practice is that they tend to "not align well with existing powerful professional communities" (p. 190). Barriers and boundaries are important to explore in investigations of how knowledge from different domains are combined to form innovations.

Some studies look into translational medicine as examples of knowledge integration practices that often occur bottom-up and in an incremental fashion (Hopkins, 2006; Lander \& Atkinson-Grosjean, 2011; Evans \& Scarbrough, 2014). Evans \& Scarbrough (2014) highlight that bridging science and clinical practice is but one approach utilized by translational teams. In their study of teams involved in translational research initiatives in the UK, they find another approach, which they label "blurring". Here translation occurs as a "continuous processes situated within routine, day to day practices", and translation or knowledge integration occurs through multiple small steps at the micro-level, which 
over time might lead to major transformation. This perspective is based on the assumption that translational processes take a long time and can only be observed after a considerable time period.

\section{Research propositions}

Summing up, the literature portrays innovation in medicine as highly dependent upon integration of multiple sources of knowledge. In this context, knowledge is distributed across multiple public and private organizations and what we have termed domains. Particular attention has been given to three domains: life science research on the causes and characteristics of disease, the clinic as a setting for observation and treatment of patients, and the firm as a main instrument of commercialization. There is a high degree of specialization as innovation depends on long-term investments and heterogeneous knowledge bases. The medical context is also characterized by multiple bridges across these domains, partly institutionalized by multi-organizational arrangements and purpose-built organizations such as academic medical centers, and also through the flow of individuals, cross-disciplinary teams and widespread networks that connect health care and scientific domains. Research facilities and tools, medical problems and patients are important boundary objects that connect and become the core of micro-innovation systems.

We argue that this setting is highly relevant for exploring issues of knowledge integration and innovation, even though the literature on these topics often addresses relationships between firms only. This literature argues that integrating diverse sources of knowledge is necessary for innovation, but also challenging. Carlile (2004) and Hansen (1999), among others, argue that significant differences in knowledge require complex processes of transfer and translation. Studies of industries or technology domains where innovation occurs frequently, and where innovation requires a high degree of specialized competencies and resources, have found that they develop a wide range of collaborative ties. Organizations and individuals that connect disparate areas of competencies have been found to innovate more often. Studies within life science (Zucker \& Darby, 1996; Hess \& Rothaermel, 2011) show that relatively few individuals contribute to a large number of inventions. Moreover, these individuals are positioned in such a way that they span or connect different domains (Ahuja, 2000; Hargadon \& Sutton, 1997).

In this article, we draw upon the insights from the literature to empirically investigate the importance of integration of knowledge across domains for medical innovation. To do so, we will look at the interaction between hospitals and universities in innovation, the form it takes and the practices that facilitate integration across domains. The main proposition that guides this research is that interaction between hospitals and academic research units is positive for generating innovations in the medical domain. Moreover, the literature on medical innovations underscores that innovation processes are lengthy and require multiple small steps and wide networks between individual and groups. A second 
proposition is that knowledge integration must become widespread and institutionalized before it results in an increased capability in generating innovations.

To analyze these propositions, we investigate empirical indications of hospital and university interaction in the generation of innovations. More specifically, we look at the patterns and frequency of co-invention activities between hospital and university staff. By investigating evidence for coinvention, we explore whether new product ideas emerge from teams or individuals within organizations, or from teams that straddle organizational boundaries. We treat cross-organizational coinvention as a proxy for knowledge integration in the process of generating innovations. We thereby assume that teams of inventors from different domains are set up to integrate various kinds of knowledge. Moreover, we assume that efforts to invent, as indicated by the submission of an idea for a new invention, is a useful proxy for early-phase innovation activities. We are aware that quite a number of the ideas for new inventions that are included in the sample will not become innovations (defined as new products, processes or services that have reached the market or have been implemented somewhere) and that many of them might represent ideas that eventually will fail. Since we are interested in understanding the process of generating novelty, and not only the preconditions for successful inventions that make it to the market, ideas for new inventions represent a rich and available data source that is easily obtainable and can be used for understanding the process of generating novelty (Ali \& Gittelman, 2016).

\section{Data and methodology}

The article studies early stage research-based inventions, using a dataset of individuals involved in generating new product ideas within life-sciences. The dataset is constructed from disclosures of inventions (DOFIs) to the joint Technology Transfer Office (TTO) of the two largest R\&D performing organizations within life science in Norway - the University of Oslo and South-Eastern Regional Health Authority in Norway. The health authority is the owner of eleven public and five private noncommercial hospitals organized as trusts, and $R \& D$ for about 220 million euros annually is performed in the organization. The University of Oslo is the country's largest academic institution. More than half of the R\&D within life science and medicine in Norway is performed by the organizations we study, with a particular high concentration of activities in the Oslo University Hospital (40 percent of all national health related $R \& D)$. With a high concentration of life science and health related $R \& D$ in the region, we assume that the organizations are also responsible for the major part of the research based innovation activities within life science and medicine in the country. As a consequence, we assume that a large share of ideas for new inventions within life science that emerged in Norway in this time interval were disclosed to the joint TTO and are contained within our database. 
In total, the database contains information about 165 ideas for potential innovations classified as life science and submitted by academic and hospital employees in the period 2009 to 2011. The DOFI database contains information about disclosed ideas that might represent an invention - or at least that the submitter assumes that the idea is novel and has a potential for application. Most of the ideas will not turn into patents and few will have a commercial output such as a licensing agreement ${ }^{1}$. DOFI data is therefore not a good source for exploring successful commercialization processes (Ali \& Gittelman, 2016), but it contains a lot of information about activities and about the individuals involved in novelty generation processes, and this type of data therefore fits our purpose.

The dataset contains information about all individuals that have been involved in submitting one or more ideas for an invention and are employed at the hospitals or universities in the region in the period 2009-2011. In total, the database contains information about 298 individuals. Relatively little information about the individuals was registered by the TTO in the database - only names and place of employment for the lead inventor at the time the invention was disclosed. We collected additional information about all individual inventors as well as data on the organizations in which the individuals were employed to be able to analyze the settings from which inventive ideas emerge (see Table 1 for an overview of variables and data sources).Table 1 about hereFor some variables, we had to use multiple sources of information to gain information about all individuals. We started with the information contained in the DOFI database, then worked stepwise to collect publicly available information about each individual. The most important additional data sources were information about individuals from employee registries (which in addition to basic information often contained CVs) and national databases for scientific publications (it is mandatory for Norwegian researchers to register their publications in a national database). We have also used the public tax registry and a publicly available registry of individuals with commercial roles (ownership in limited companies and appointments in board of directors in such companies). In a few cases, we used LinkedIn to confirm identification of the individuals in the database.

In the data collection and analysis, we have made the decision criteria for collecting data transparent and through this more reliable. We have also relied on multiple data sources to corroborate the data and through this increase the validity of the analysis. A possible threat to the reliability is that we have collected information based on inventor names with possible "false positives". However, name combined with workplace information and other data meant that we were confident that we were able to verify in most cases that we were collecting information about the right individuals. A few individuals that we were uncertain about after recursive attempts at confirmation were omitted from

\footnotetext{
${ }^{1}$ The dataset does, however, include information about the decision made by the TTO to develop the idea further, patents generated and licenses attached to each idea. The data on the performance of the disclosed ideas is not included in our analysis.
} 
the analysis. A second challenge to the reliability and validity of the analysis is that the individuals may have changed place of employment after filing the DOFI. This is important because we use place of employment as a proxy for the location from which the invention originated. In the cases where place of employment was not found in the DOFI database, the coding of origin for the invention was based on most recent employment data, with limited possibility for controlling the accuracy. We have no reason to believe, however, that the data contain systematic miscoding in such a way that one site is systematically being over- or underrepresented. Still, this weakness in reliability means that interpretations of the findings must be made with care. Further research is needed to test the methods and to collect data on larger samples, would increase the reliability and validity of the research.

In addition to the degree and type of interaction between hospitals and universities in invention, we look at whether individuals involved in inventive activities in hospitals and universities resemble each other in terms of occupational categories, educational background, commercial and scientific productivity, and the kinds of inventions they had contributed to. We investigate whether there are distinct communities involved in innovation work in hospitals and universities, or whether we see blurring communities (Evans \& Scarbrough, 2007) where individuals resemble each other regardless of place of employment. For these purposes, the data was analyzed mainly to generate descriptions and comparisons of groups, by use of correlational analysis and analysis of significant group differences.

We also wanted to understand at a more detailed level why hospital-university connections matter for the ability to generate novelty and how the connections are utilized in such processes. We therefore selected the most active inventors from the sample to be interviewed, as we assumed they would be the most experienced inventors in the sample. As inventive activity among academics and hospital staff is highly skewed, the interviewees have been involved in a considerable share of the inventive ideas contained in the database. All inventors selected for interviews had been part of submitting at least three DOFIs in the period 2009-2011. 20 individuals were contacted; thirteen of them were employed in the hospital and seven in the university. Ten of them agreed to be interviewed.

In the interviews, the informants were asked questions about their involvement in inventive work, focusing on the particular inventions we knew they had contributed to. Most of the informants had been involved in a larger number of invention processes than the ones registered in the DOFI database, and as a result we got information about a larger number of inventions than the ones we initially contacted them to discuss. We asked them several questions about the inventions, how they had been developed and the results of the inventive process. Then we asked several questions about to what extent and how they had interacted with clinical or academic units (depending on the site) in the inventive process, and what they gained from interacting, as well as potential challenges and practices set up to facilitate knowledge interaction. 


\section{Invention processes in life science: a result of multi-organizational}

\section{collaboration?}

As mentioned the DOFI database contains information about 165 disclosed ideas submitted by 296 individuals. Slightly more than half of the inventors work in hospitals, 40 percent at universities, and 7 percent in firms. Among the individuals listed as the lead inventors, 50 percent are employed in the hospital sector and 50 percent in the university sector. 30 percent of the lead inventors are employed both at the hospital and university, indicating that boundary spanners are important for generation of new ideas in life science.

The relative share of hospital inventions and academic inventions differs between product segments, but these differences are not as clear as expected. As can be seen in Figure 1, inventive ideas are mainly found within four product segments: Diagnostics, medical devices, research methods and therapeutics, with less than 5 ideas in each of the remaining categories. The product taxonomy has been created by the Technology Transfer Office. Hospital staff has a larger share of inventive ideas in medical devices, and university staff a larger share within therapeutics and new therapeutic components, as expected. Hospital staff and university staff have a relatively equal share of inventions within diagnostics and research tools (Figure 1).

\section{Insert figure 1 about here}

Relatively few individuals are as serial innovators (i.e. contributing to more than one disclosure). 71 percent have only contributed to one DOFI, while 19 percent have contributed to at least two DOFIs in period and 10 percent to more than two. A very small group of people (29) has contributed to multiple new ideas. The highest number of ideas registered on one person is 14. Comparing universities and hospitals in this respect, we see that 66 percent of inventors from the hospital have submitted only one DOFI, while 75 percent of university employees occur only once in the dataset. 22 percent of the hospital staff has supplied two DOFIs and 11 percent three or more. The corresponding figures from the university are 15 and 10 percent. The difference in the number of ideas submitted by hospital and university staff is, however, not statistically significant, and the average number of DOFIs for the two groups is identical (1.56).

40 per cent of the DOFIs have been submitted by individuals or groups only from the university or only from a hospital, while 60 percent have been submitted by groups representing multiple institutions. Of the latter, 37 percent are from teams representing units in both universities and hospitals, and 23 percent are from individuals and groups with multiple affiliations (employed at the same time in a hospital and the university). 
Multi-institutional collaboration about idea generation is most common in the product segments diagnostics, research tools and therapeutics, and less common in medical devices. Multi-institutional collaboration is equally common for inventions that emerge from clinical units in hospitals as from scientific/university units, but it is more common for hospital-based inventions to emerge from individuals that have dual affiliation positions. These are mainly individuals employed in adjunct positions in universities in addition to holding a hospital job (Figure 2).Insert figure 2 about here

\section{A community that straddles institutional boundaries?}

The ideas reported as disclosures of inventions in our life science data are potential product innovations that in some cases can be protected by patents, and are often connected to research activities. Consequently, we expect personnel in research positions and with research competencies to be overrepresented also among hospital staff. To investigate further we looked at current occupational positions, level of education, and number of scientific publications for all inventors.

Employees in many different occupational categories are involved in activities that lead to potential innovations. Professors (27 percent), researchers (16 percent) and medical doctors/physicians (14 percent) are the groups most often involved in such activities. Among hospital employees, medical doctors are the most frequently occurring group ( 25 percent of the hospital staff), followed by professors and researchers (17 and 16 percent). Among the university employees, professors dominate (44 percent).

Overall, more than 70 percent of the sample have a $\mathrm{PhD}$ degree or equivalent. Many of those with a $\mathrm{PhD}$ are also trained medical doctors. Among those without a doctoral degree, 10 percent have a master's degree and 10 percent have a medical degree; 2 percent are trained nurses. There are no significant differences between hospitals and universities in the relative proportion of $\mathrm{PhD}$ holders among inventors (72 percent among hospital employees and 77 percent among university employees).

In both groups, formal research competences are strong, as evidenced by their scientific publications (Figure 3). There is, as expected, a huge variation in the number of publications (from 0 to 551!); the average number of publications is 67 and the standard deviation is 97. Divided into groups, we see that the majority have at least one publication to their name ( 84 percent), and that 40 per cent of the inventors have more than 50 publications. Figure 3 indicates that there is little difference between the hospital and university employees' research productivity. Insert figure 3 about hereWe have also looked at the relationship between the number of reported ideas and research productivity. There is a weak but statistically significant correlation between the number of scientific publications and the number of disclosures people have contributed to ( 0.24 at the .01 level). Among those who participated in two or more disclosures ( 29 persons), half (15 people) have more than 100 scientific 
publications. Still, a significant number of ideas are disclosed by younger personnel with weak publication records (so far) from both the university and hospitals.

The literature in this field highlights how cooperation between clinical settings and academic settings is important for innovation in life science. Most studies have looked at cooperation in later development phases (Blume, 1992; Rosenberg et al., 1995; Gittelman, 2016) of medical technology or medical practice, while few have emphasized collaboration in the invention/idea generation stage (Alkærsig \& Valentin, 2016; Ali \& Gittelman 2016). Our data shows that cooperation between the clinic and academia is important in the idea development stage as well - because innovative ideas often come from groups ( 3 out of 4 ) and because 60 percent of new ideas emerge from individuals or groups that have more than one institutional affiliation. The highest degree of cooperation is found in the areas of diagnostics, research methods and the development of new therapeutic components. Overall, a high proportion of ideas clearly emerge out of environments that cut across the clinic and academia. Individual level data on positions, formal competencies, and practical research experience support this claim as well. Both among the hospital employees and the university employees we find that inventors have high formal and practical research qualifications, and that there are no significant differences at the individual level between the two groups. This indicates that individuals and teams involved in invention processes in life science represent a community that straddles institutional boundaries. How this occurs and why it is important for innovation is explored further in the next section.

\section{Knowledge integration practices}

To explore why collaboration between hospitals and universities is widespread in life science invention processes, we carried out interviews with prolific inventors as outlined in the methodology section. The informants highlighted the cross-disciplinary nature of their scientific work, an aspect particularly important in the context of developing new ideas with an innovation potential. A professor of biophysics said, "Inventions are usually spin-offs from research activity. In my area [biophysics], promising research is carried out through collaboration between multiple groups. Physicist are good at measuring things, chemists can make the molecules and drugs, biologists are experts on the composition of the cells and cancer researchers and oncologists measure and monitor tumor growth. We complement each other." Other informants described similar patterns.

Most of the informants work in areas that are cross-disciplinary in nature and which operate at the intersection between basic and applied or clinical research, as indicated by the following statements: "My group works on signal regulation in organisms such as hormones ... which is a generic discipline with broad [practical] relevance" (Professor, biotechnology). "Antibodies is a special area, it is both an academic field and a category of drugs which is sold and used every day" (Professor, biology). 
This cross-disciplinary work mode includes clinical groups and units as well, and access to clinicians was not a problem for the informants. A biotechnology professor said that his center had more than 100 collaboration agreements with various clinical groups, "literally everyone nationally" as well as a large number of foreign ones. A biology professor stated: "In our setting at the intersection of the university and the hospital, there are numerous formal collaboration agreements between various groups and organizations. This is just there and something we can exploit, which we do." Another informant, a professor in biophysics, said that his group was "swimming in clinical environments. The relationship is very close. We talk on a daily basis and I supervise a large number of medical doctors." Finally, a professor of medicine stated even though there is a high degree of interaction across scientific and clinical domains, there is still a clear distribution of labor; different units are responsible for different stages in the innovation process. He explained the nature of his work and integration with clinical facilities in the following way: "We do basic research that has a broad area of application. We are interested in exploring different areas of potential use of the basic technology, and mainly collaborate with clinical facilities in clinical testing stages. In these stages we collaborate with many different clinical units.”

Inventors working in hospitals described a similar need; for both idea generation and testing knowledge and other resources from technological or scientific units in the universities or in small, specialized technology firms is needed. In the words of a medical doctor in charge of a hospital unit that develops and tests new medical technologies, "Access to technological knowledge is really important. We have dense external networks to technical and natural science research communities, such as in micro technology, informatics and robotics."

Based on information collected through the interviews, we see five different mechanisms or practices leading to integration of academia and the clinic. First, some work in cross-domain teams, especially within research hospitals, where scientists and clinicians are members of the same teams. Second, there are numerous formal collaboration agreements that facilitate collaboration and define the practical terms of it including cost structures for externally funded activities and ownership to research results. Third, there are personal/informal networks that extend across domains. Fourth, we observe a number of dual affiliations among the interviewees. This has both a personal side, such as belonging to two different organizations and for some having trained as medical doctor, and an organizational side when laboratories and other facilities are shared between the university and the hospital. Finally, multiple boundary objects are important for inter-organizational collaboration and knowledge integration. In the interviews, particularly scientific facilities and tools, patient samples and clinical trials serve to integrate groups, and shared resources and knowledge emerge around these objects and areas of practice. 
Another way at looking at knowledge integration practices is to look at the reasons for why collaboration is important and the role knowledge integration plays for innovation, seen from the perspective of the informants. In general, interaction between clinical and scientific units was hailed as essential for innovation but in some cases also for research: "Cross-disciplinary approaches are very important and the ability to think outside of the box. For us (biologists working in a cancer research facility in the hospital) collaboration with pathologists is very important. We need access to patient samples (tumors) and the collection of a large biobank has been decisive for both research and innovation activities" (Hospital Researcher). A biology professor said that in some cases clinical collaboration is essential, or "the innovation will simply not happen". She stated that clinicians' expertise is necessary for collecting the right samples in the right manner: "If you need tissue samples, you need clinical expertise. If you work on coeliac disease, you need the patient to eat bread for three days and then a lot of specific tests to be carried out, and the clinicians are the ones who know the patients, who can convince them to take part and do the correct tests afterwards. This is absolutely necessary."

A biotechnology professor also highlighted the "close-to-market" aspects and the technical aspects of involving clinicians in inventive work: "It contributes with a market calibration; [clinicians] can help us understand what the problems are, what the patients need, what it is possible to administer [of a drug]; what the utility value of something is. Clinicians are not least important when we have a therapy product with specific indications in humans. How should you measure effects? Effect studies in humans are designed to try to get the answer you are looking for. It costs so much money if you get it wrong, and then the project may be stopped although the idea may still be good. Inclusion criteria and other things are so important ... and clinicians have a daily work where they can see what is relevant."

In addition to the access to tools and methodologies and complementary areas of knowledge described earlier, this section has highlighted two main benefits from interaction between academics and clinicians. First, clinical experience provides knowledge about relevant problems and user needs as well as uses of existing solutions and their shortcomings. This narrows the search scope and helps attune the innovation activities to patients in a better way. Second, clinical collaboration allows access to biobanks, patient material, and clinical knowledge used in design of clinical studies and sites for early-phase clinical experimentation. This can provide early validation of ideas but seems even more important in later stages of larger-scale testing. Whether clinical-academic interaction is important in the idea generation/early phase or not may differ e.g. according to the type of product/process in question, scientific specialty, involvement of firms and more. Quality control of concepts and prototypes as well as testing procedures was also seen as an important reason for involving clinical personnel in hospitals. Some of these procedures are very complicated and rely on tacit knowledge and expertise that scientific units rarely possess. Failure to apply stringent testing procedures may 
constitute a problem for later commercialization, e.g. if approval criteria of drug regulatory agencies demand specific types of documentation and testing.

\section{Discussion}

The analysis has provided new information about the people and communities that are involved in developing ideas for inventions in medicine, highlighting practices that promote knowledge interaction and integration across organizations. Existing literature argues that combination of different but complementary sources of knowledge is important for innovation, but challenging to archive in practice (Tell, 2011; Carlile, 2004). In the healthcare context, innovation activities occur in clinical and scientific units in hospitals, in universities and in firms, and actors within this heterogeneous system are involved in generating ideas, inventions and innovations in the form of new products and services. Empirical and theoretical writings on innovation in medicine and health indicates that innovation in this sector relies on heterogeneous and decentralized knowledge bases, and that interaction across different sources of knowledge is particularly important (Mina, 2009; Morlacchi \& Nelson, 2011).

To contribute to this literature, the present study has looked at interaction between universities and hospitals and whether this is conducive for generating new ideas for inventions, as assumed by the literature. We have focused on interaction about inventive activities, based on a unique dataset of disclosed ideas for new inventions. The results of our study indicate that interaction between scientific and clinical domains is common and important for innovations in medicine and health, in accordance with the propositions. More than half of the ideas in the disclosures database emerge from teams representing multiple institutions that span scientific and clinical units or from individuals with multiple institutional affiliations. Collaborating across scientific and clinical domains is clearly an institutionalized practice in medical research and innovation communities. There are formal collaboration agreements between organizations; individuals have personal networks expanding into other domains and sectors; individuals have dual affiliation and/or work in joint facilities or labs, and research teams contain members that have heterogeneous backgrounds. There are also multiple examples of boundary objects that connect the domains including tools, methods and research materials, patients, and tissue samples. In line with our second proposition, the data indicates that practices for knowledge integration between hospitals and universities are widespread and institutionalized, and that the community of clinical and scientific research units has well-established and effective means to carry out research and generate ideas for new potential innovations.

Also in line with prior research (Nelson et al., 2011; Morlacchi \& Nelson, 2011) we find that clinical units are important in the idea generation phase and not only in testing and post-introduction improvement. Hospital units in the sample generate about half of the new ideas and are heavily 
involved in the ideas that emerge from scientific units. Lander \& Atkinson-Grosjean (2011) argues that the integration between scientific and clinical environments is particularly important for the noncommercial or "translational pathway" of life science research. Our analysis, as well as research by Ali \& Gittelman (2016) indicates that integration between clinical and scientific competencies is also important in the "commercialization pathway". However, this result needs substantial further research, utilizing other data sources than disclosed inventions.

We have looked into individual characteristics to see whether people active in innovation in life science are similar of different. Similarity between people employed in different organizations would indicate the existence of an innovation community that straddles boundaries between different domains (Evans \& Scarbrough, 2007). Our data shows that there is surprisingly little difference between the hospital inventors and university-based inventors on individual-level characteristics. Previous research on university employees has found a similar pattern to ours in terms of the kind of scientists that are the most active innovators (van Looy et al., 2004; Gulbrandsen \& Smeby, 2005; Geuna \& Nesta, 2006). Our data also shows similar patterns among hospital staff; it is interesting that similar accumulation of advantage or quality effects may be at play here.

\section{Implications and conclusion}

Our empirical study from the medical innovation context illustrates how important knowledge interaction is for inventive activities. Moreover, the study illustrates how pervasive these links are in this specific context. Most studies of knowledge integration and its role in innovation focus on firms and networks between firms. This article has illustrated the importance of investigating the role of teams and individuals in knowledge integration across organizations (Tell, 2011, Andersson \& Berggren, 2011, Carlile, 2004). At the same time, our study indicates that networks exist at several levels, between organizations, between professions and groups, and at the individual levels. Networks are inherently interconnected. One implication is that studies of knowledge integration should attempt to carry out multilevel analyses, where ties between organizations and individuals are investigated within the same context (Moliterno \& Mahony, 2011). In this study, we clearly see that multiple networks are layered on top of each other, and together they make a deeply embedded structure of contacts that seems to be vital for generating novelty in a highly specialized and knowledge intensive context.

This study has several limitations, and should be followed by more in-depth empirical research. Data was collected from a limited empirical site (one region in Norway) and focused on a limited kind of inventive activities - generation of new product ideas. The kinds of innovation processes we have looked at are often based on research and a high degree of novelty is required for the ideas to be accepted and followed up, and consequently interaction between scientific and clinical environments should be expected. A similar result as ours may not have been obtained if the study focused on a 
broader range of innovation processes, or other sets of actors or sectors. Furthermore, limited data was available at the time this study was conducted. A longer time series and more detailed individual level data would be preferable, combined with data on the outcomes of the inventive processes. Further research should take these shortcomings into account, and preferably collect data from multiple sites to avoid selecting environments where interactions between sites are particularly common.

Highlighting challenges involved in collaboration between clinical and academic communities and issues connected to management of such linkages are other relevant questions for further research.

There are several practical implications for policymakers and managers of innovation work within the health care context, who may in general benefit from more systematic information about inventive activity in life sciences and medicine. We have seen that this activity is very often the result of collaboration across organizational borders, especially within important areas such as diagnostics, research tools and therapeutics. Managers responsible for innovation in medicine and health care should ensure that the infrastructure for collaboration is in place. A central element here are institutional framework agreements that make it easy to set up new cooperative ventures and give different actors access to laboratory space, specific equipment, and more. Framework agreements signal a top-level commitment to collaboration and give clear and consistent rules for difficult areas such as funding of joint work, shared space, equipment, and intellectual property rights. The latter issue can represent a potential problem, as hospitals and universities operate with slightly different IPR-regimes. Therefore a joint technology transfer office, as the units in this study share, is likely to be very positive for aligning regulations and also for creation of common incentives to cooperate. Shared facilities across scientific and clinical units, including multi-institutional research teams and positions with double affiliations, are other initiatives that is likely to have a positive influence on inventive activity. Meetings or other forms of interaction where the needs and challenges of patients and clinical staff is clearly communicated to scientists may also have an effect, as understanding users' needs seems to be as important for successful innovation in health care (Lettl et al., 2006) as it is in general (von Hippel, 2009) .

\section{Acknowledgements}

This article is based on the STILS research project funded by a grant from the Norwegian Research Council, under the program activity FORFI, and a grant from the Health Region South-East in Norway. Their financial support is gratefully acknowledged. We also would like to thank Inven2 for supplying data, Ole Kristian Berggren for additional assistance in collecting data and all the informants that took part in the interview study. The insightful comments made by the participants at the annual WOMI workshops and the reviewers and editors of EJIM are also acknowledged. Any mistakes and omissions remain the sole responsibility of the authors. 


\section{References}

Ahuja, G. (2000). Collaboration networks, structural holes, and innovation: A longitudinal study. Administrative science quarterly, 45(3), 425-455.

Ali, A. \& Gittelman, M.(2016). Research paradigms and useful inventions in medicine: Patents and licensing by teams of clinical and basic scientists in Academic Medical Centers. Research Policy (2016).

Alkersig, L. \& Valentin, F. (2016). Effects on medical innovation of combining academic and clinical expertise. (Unpublished manuscript).

Andersson, H. \& Berggren, C. (2011). Inventors as innovators and knowledge integrators. Knowledge Integration and Innovation: Critical challenges facing international technology-based firms. Edited by Berggren, C., Bergek, A., Bengtsson, L. \& Söderlund, J Oxford: Oxford University Press

Blume, S. S. (1992). Insight and industry: on the dynamics of technological change in medicine. Cambridge, Mass., MIT Press.

Carlile, P. R. (2004). Transferring, translating and transforming: An integrative framework for managing knowledge across boundaries. Organization science. 15(5), 555-568

Consoli, D. and A. Mina (2009). An evolutionary perspective on health innovation systems. Journal of Evolutionary Economics 19(2): 297-319.

Evans, S., \& Scarbrough, H. (2014). Supporting knowledge translation through collaborative translational research initiatives: 'Bridging'versus 'blurring'boundary-spanning approaches in the UK CLAHRC initiative. Social Science \& Medicine, 106, 119-127.

Gittelman, M. (2016). The revolution re-visited: Clinical and genetics research paradigms and the productivity paradox in drug discovery. Research Policy.

Hansen, M. T. (1999). The search-transfer problem: The role of weak ties in sharing knowledge across organization subunits. Administrative science quarterly, 44(1), 82-111.

Hargadon, A., \& Sutton, R. I. (1997). Technology brokering and innovation in a product development firm. Administrative science quarterly, 716-749.

Hess, A. M., \& Rothaermel, F. T. (2011). When are assets complementary? Star scientists, strategic alliances, and innovation in the pharmaceutical industry. Strategic Management Journal, 32(8), 895-909.

Hicks, D. and J. S. Katz (1996). Hospitals: the hidden research system. Science and Public Policy 23(5): 297-304.

Hipple, Eric von (2005). Democratizing Innovation. Cambridge, Massachusetts: MIT Press.

Hopkins, M. M. (2006). The hidden research system: the evolution of cytogenetic testing in the National Health Service. Science as Culture 15(3): 253-276.

Lander, B. (2013). Sectoral collaboration in biomedical research and development. Scientometrics, 94(1):343-357 
Lander, B. and J. Atkinson-Grosjean (2011). Translational science and the hidden research system in universities and academic hospitals: A case study. Social Science \& Medicine 72(4): 537-544.

Lettl, C., Herstatt, C., \& Gemuenden, H. G. (2006). Users' contributions to radical innovation: evidence from four cases in the field of medical equipment technology. R\&D Management, 36(3), 251-272.

Merito, M. and A. Bonaccorsi (2007). Co-evolution of physical and social technologies in clinical practice: The case of HIV treatments. Research Policy 36(7): 1070-1087.

Metcalfe, J. S., et al. (2005). Emergent innovation systems and the delivery of clinical services: The case of intra-ocular lenses. Research Policy 34(9): 1283-1304.

Mina, A. (2009). The emergence of new knowledge, market evolution and the dynamics of microinnovation systems. Economics of Innovation and New Technology, 18(5): 447-466.

Morlacchi, P. and R. R. Nelson (2011). How medical practice evolves: Learning to treat failing hearts with an implantable device. Research Policy 40(4): 511-525.

Moliterno, T.P., \& Mahony, D.M. (2011). Network theory of organization: A multilevel approach. Journal of Management, 37 (2), 443-467

Nelson, R. R., et al. (2011). How medical know-how progresses. Research Policy 40(10): 1339-1344.

Ramlogan, R., et al. (2007). Networks of knowledge: The distributed nature of medical innovation. Scientometrics 70(2): 459-489.

Robertson, M. (2007). Translating breakthroughs in genetics into biomedical innovation: The case of UK genetic knowledge parks. Technology Analysis \& Strategic Management, 19(2), 189-204.

Rosenberg, N. (2009). Some critical episodes in the progress of medical innovation: An AngloAmerican perspective. Research Policy 38(2): 234-242.

Rosenberg, N., et al. (1995). Sources of medical technology: universities and industry. Washington, D.C., National Academy Press.

Schumpeter, J. A. (1934). The theory of economic development: An inquiry into profits, capital, credit, interest, and the business cycle (Vol. 55). Transaction publishers.

Tell, F. (2011). Knowledge integration and innovation: a survey of the field. Knowledge Integration and Innovation: Critical challenges facing international technology-based firms, 20-58. Oxford: Oxford University Press

Thune, T., \& Mina, A. (2016). Hospitals as innovators in the health-care system: A literature review and research agenda. Research Policy.

Zucker, L. G., \& Darby, M. R. (1996). Star scientists and institutional transformation: Patterns of invention and innovation in the formation of the biotechnology industry. Proceedings of the National Academy of Sciences, 93(23), 12709-12716. 


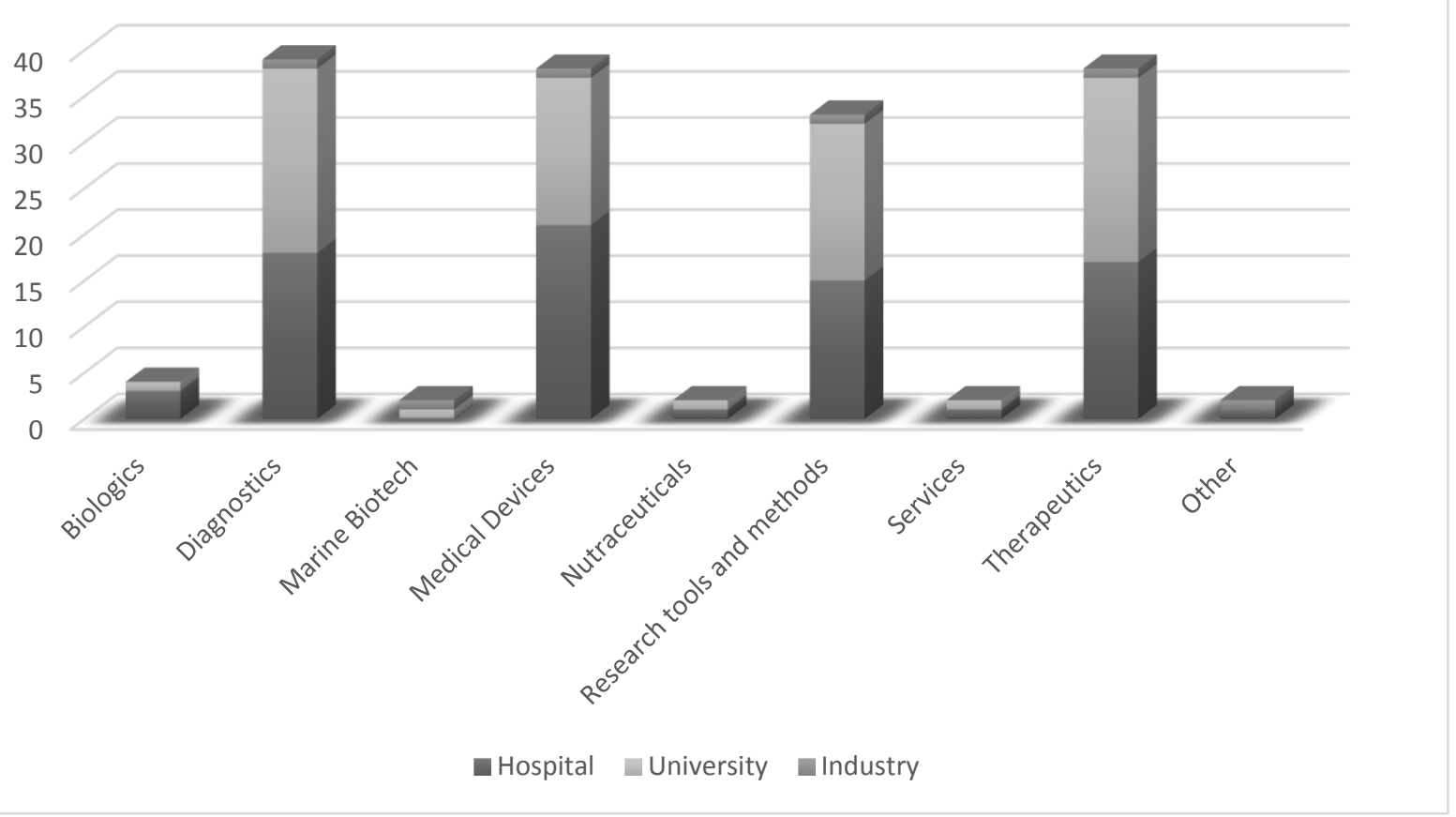

Figure 1: Distribution of ideas by product class and lead inventors' main institutional affiliation $(N=165)$

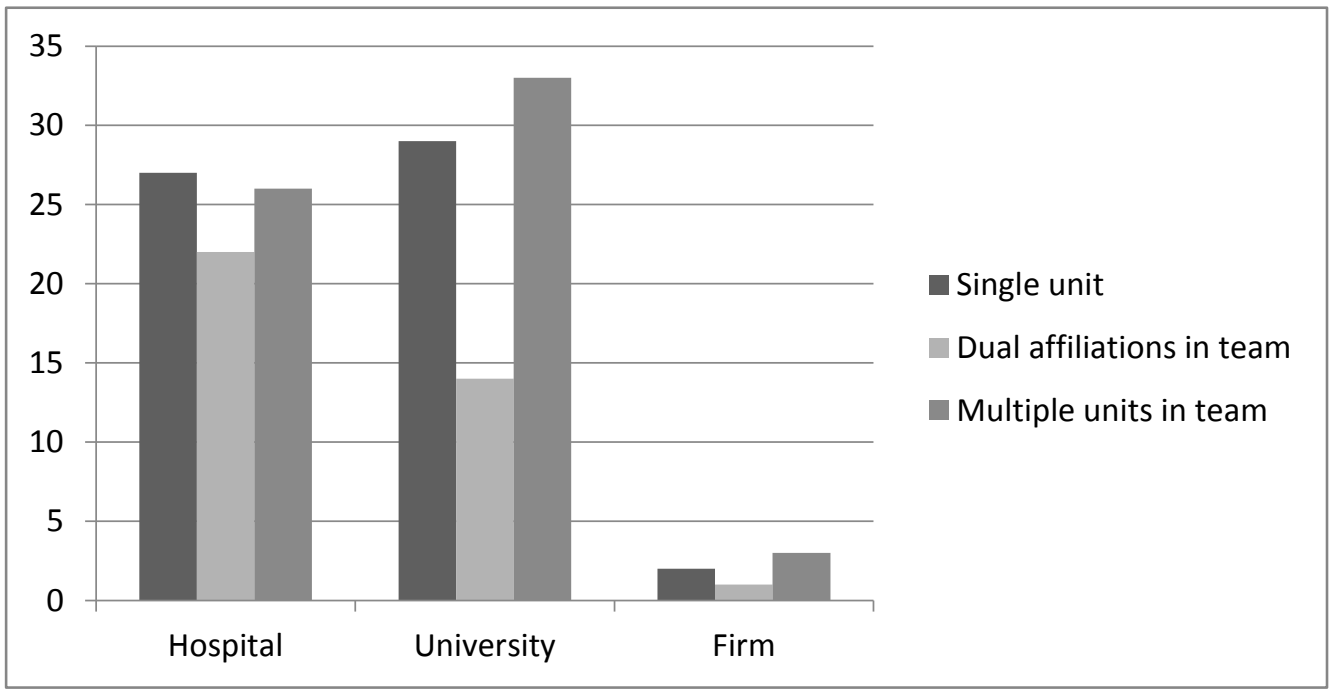

Figure 2: Collaboration on idea generation in hospitals, universities and firms $(N=165)$ 


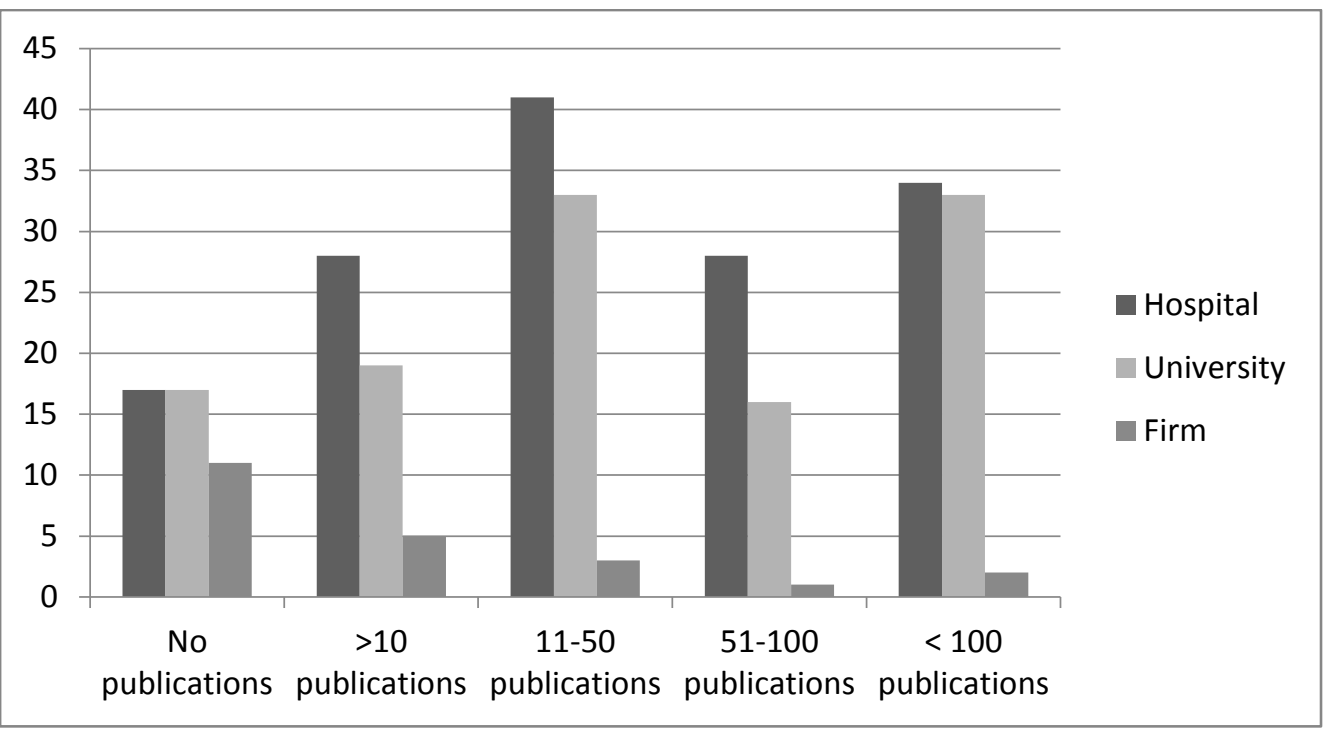

Figure 3: Research productivity among inventors from firms, universities and hospitals ( $N=287)$ 